\title{
Dark Matter after LHC Run I: Clues to Unification
}

\author{
Keith A. Olive ${ }^{1, a}$ \\ ${ }^{1}$ William I. Fine Theoretical Physics Institute, School of Physics and Astronomy, \\ University of Minnesota, Minneapolis, MN 55455, USA
}

\begin{abstract}
After the results of Run I, can we still 'guarantee' the discovery of supersymmetry at the LHC? It is shown that viable dark matter models in CMSSM-like models tend to lie in strips (co-annihilation, funnel, focus point). The role of grand unification in constructing supersymmetric models is discussed and it is argued that non-supersymmetric GUTs such as $\mathrm{SO}(10)$ may provide solutions to many of the standard problems addressed by supersymmetry.
\end{abstract}

\section{Introduction}

Among the motivations for supersymmetry is the theory's ability to provide gauge coupling unification [1] and address the gauge hierarchy problem [2]. It is well known that the additional fields predicted in minimal supersymmetric standard model (MSSM), if present at low energy, alter the running of the gauge couplings as shown in Figure 1. In order to ensure that the proton is long-lived, it is common to impose $R$-parity defined in terms of baryon number, lepton number and spin as $(-1)^{3 B+L+2 s} . R$ parity limits the models to include only those interactions which are direct supersymmetric analogues of Standard Model (SM) processes. As an additional consequence, supersymmetric models with $R$ parity predict the existence of a stable particle which can be a dark matter candidate [3].

Many models of supersymmetry breaking (such as gravity mediation [4]) predict universalities among the supersymmetry breaking parameters. For example, at some high energy input scale (usually taken to be the GUT scale), all gaugino masses take a common value, $m_{1 / 2}$, all scalar masses, $m_{0}$, and all trilinear mass terms, $A_{0}$. These three parameters, together with the ratio of the two Higgs expectation values, $\tan \beta$, defines the constrained MSSM (CMSSM). In the CMSSM, one uses the conditions derived by the minimization of the Higgs potential after radiative electroweak symmetry breaking to solve for the Higgs mixing mass, $\mu$ and the bilinear mass term $B_{0}$ (or equivalently $\mu$ and the Higgs pseudoscalar mass, $m_{A}$ ) for fixed $\tan \beta$.

As discussed later, non-supersymmetric $\mathrm{SO}(10)$ models can also naturally account for the presence of a dark matter candidate. If symmetry breaking to the SM occurs through an intermediate scale gauge group. The gauge couplings may be deflected at the intermediate scale and hence allow for gauge coupling unification as shown in Fig. 2 [5]. If the intermediate scale is broken via a $\mathbf{1 2 6}$ dimensional representation of $\mathrm{SO}(10)$, a $Z_{2}$ discrete symmetry (similar to $R$-parity) is preserved thus allowing for the existence of a stable dark matter candidate.

a e-mail: olive@umn.edu 

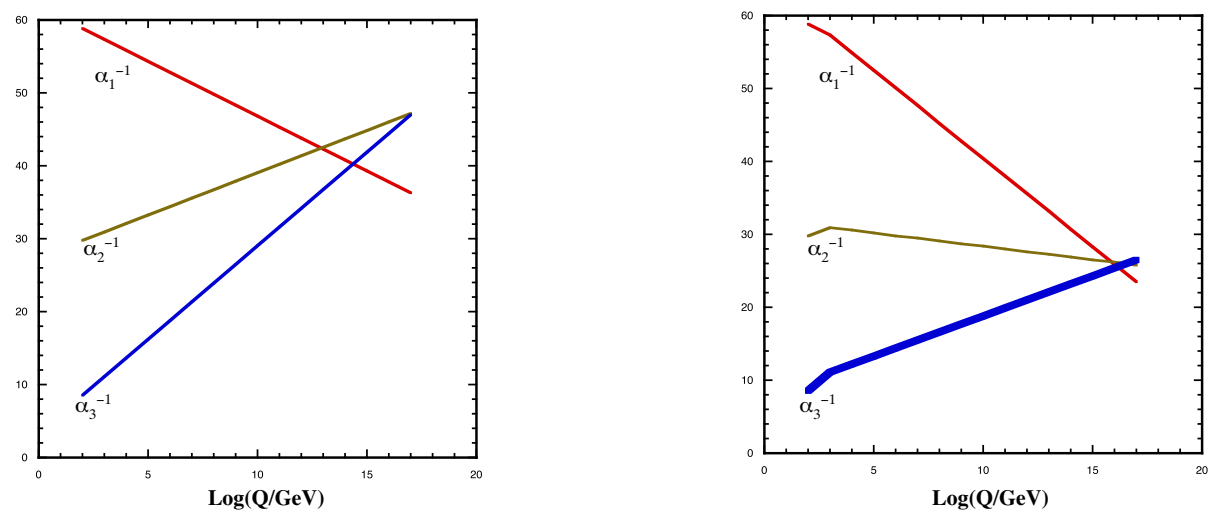

Figure 1. Running of the gauge couplings in the standard model (left) and in the MSSM (right). $\alpha_{i}=g_{i}^{2} / 4 \pi$ for each of the gauge couplings associated with $\mathrm{SU}(3)_{c}, \mathrm{SU}(2)_{L}$, and $\mathrm{U}(1)_{Y}$. Unification of the couplings becomes possible in the MSSM.

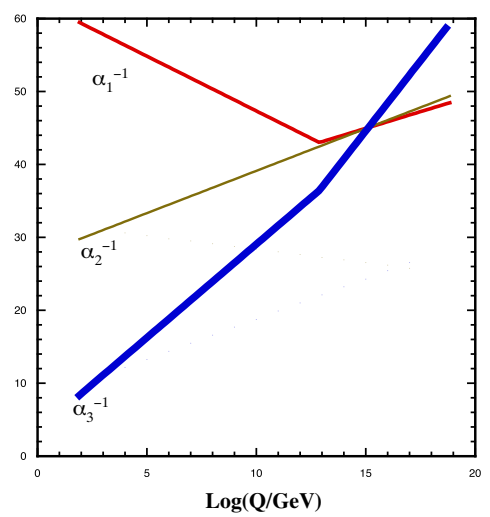

Figure 2. Running of the gauge couplings in a $\mathrm{SO}(10)$ model broken to $\mathrm{SU}(4) \times \mathrm{SU}(2)_{L} \times \mathrm{U}(1)_{R}$ at the unification scale, $M_{G U T}=1.3 \times 10^{15} \mathrm{GeV}$, which is subsequently broken to the Standard model at an intermediate scale $M_{\text {int }}=7.8 \times 10^{12} \mathrm{GeV}$.

\section{Pre-Run I}

Before Run I at the LHC, there was much anticipation for the possibility of discovering supersymmetry as supersymmetric models such as the CMSSM provided definite improvements to low energy precision phenomenology and were well within the range of the LHC. The left panel of Fig. 3 shows the results of mastercode [6] - a frequentist Markov Chain Monte Carlo analysis of low energy experimental observables in the context of supersymmetry. At each point sampled in the CMSSM, mastercode computes a set of observables and compares that to experiment, thus establishing a $\chi^{2}$ likelihood function across the parameter space. The figure shows the color coded values of $\Delta \chi^{2}$ relative to the best fit point shown by the white dot at low $m_{1 / 2}$ and low $m_{0}$. Marginalization over $A_{0}$ and $\tan \beta$ was performed to produce this $\left(m_{0}, m_{1 / 2}\right)$ plane. The best-fit CMSSM point lies at $m_{0}=60 \mathrm{GeV}$, $m_{1 / 2}=310 \mathrm{GeV}, A_{0}=130 \mathrm{GeV}, \tan \beta=11$ with $m_{h}=114.2 \mathrm{GeV}$. This was a pre-LHC prediction 

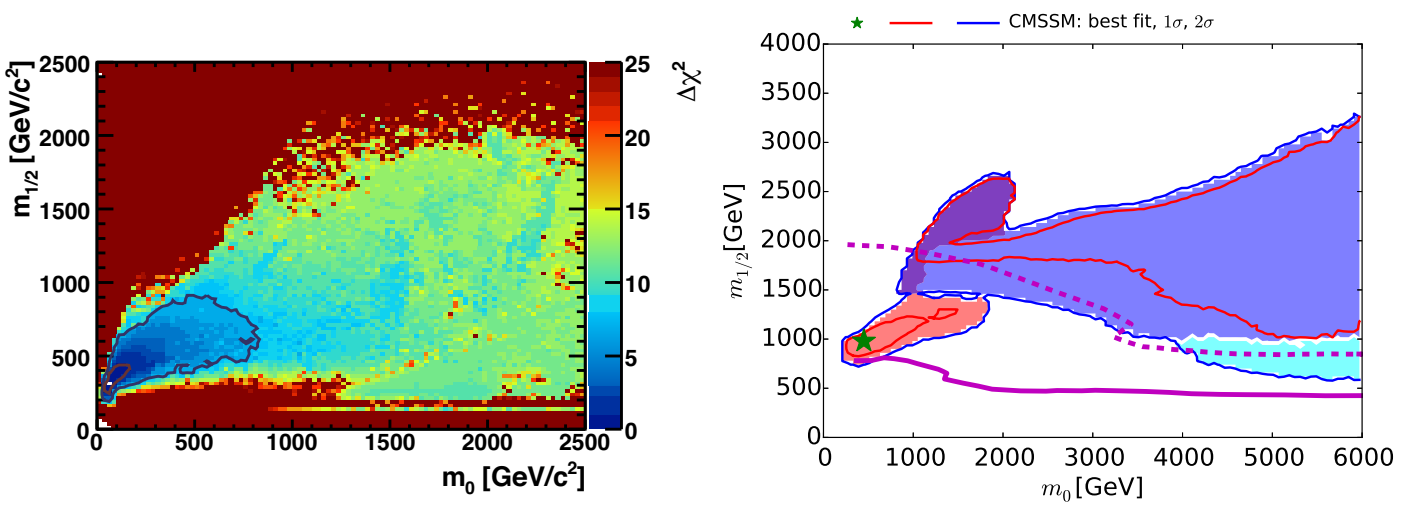

Figure 3. The $\Delta \chi^{2}$ functions in the $\left(m_{0}, m_{1 / 2}\right)$ planes for the CMSSM from a mastercode frequentist analysis. The pre-LHC result is shown in the left panel [6]. Red and blue contours correspond to $68 \%$ and $95 \%$ CL contours and the best fit point is depicted by a white dot. The post-LHC result is shown in the right panel [7] using $8 \mathrm{TeV}$ data at $20 \mathrm{fb}^{-1}$. Here the best fit point is shown by the filled star. The color of the shaded region indicates the dominant annihilation mechanism for obtained the correct relic density: stau coannihilation-pink; $A / H$ funnel-blue; focus point-cyan; and a hybrid region of stau coannihilation and funnel-purple. The solid and dashed purple curves show the run I reach and the expected run II reach at $14 \mathrm{TeV}$ at $3000 \mathrm{fb}^{-1}$ respectively. The latter corresponds approximately to the $95 \%$ CL exclusion sensitivity with $300 / \mathrm{fb}$ at $14 \mathrm{TeV}$.

and used no LHC data. The low energy observables used included $\left(g_{\mu}-2\right), M_{W}, B \rightarrow \tau v, b \rightarrow s \gamma$, the LEP limit on the Higgs mass, forward-backward asymmetries among others (for a full list of observables used see [6]). The relatively low value of $m_{h}$ was a common prediction of MSSM models [8]. A dedicated scan for the distribution of Higgs masses in the CMSSM was made in [9]. It was found that when all phenomenological constraints (with or without $\left(g_{\mu}-2\right)$ ) are included, all models yielded $m_{h} \leq 128 \mathrm{GeV}$. When $\left(g_{\mu}-2\right)$ is included, only models with $m_{h}<126 \mathrm{GeV}$ were found. Note that the scan sampled scalar and gaugino masses only out to $2 \mathrm{TeV}$.

There was equal optimism for discovering supersymmetric dark matter in direct detection experiments. The left panel of Fig. 4 displays the pre-LHC preferred range of the spin-independent DM scattering cross section $\sigma_{p}^{\mathrm{SI}}$ (calculated here assuming an optimistic $\pi$-N scattering term $\Sigma_{N}=64 \mathrm{MeV}$ ) as a function of $m_{\chi}$ [6]. The expected range of $\sigma_{p}^{\text {SI }}$ lied just below the then present experimental upper limits (solid lines) [10,11]. As one can see from the successive lower upper limits from later experiments [12-14] shown by the bands, the pre-LHC values for the elastic scattering cross section was well within reach of current experiments.

\section{Post Run I}

After run I at the LHC, the prospects for discovering supersymmetry looked very different. In the right panel of Fig. 3, the post-Run I likelihood contours in the $\left(m_{0}, m_{1 / 2}\right)$ plane [7] are shown using 8 $\mathrm{TeV}$ results at $20 \mathrm{fb}^{-1}[18]$. The best fit point based on the $8 \mathrm{TeV}$ data is shown by the filled star at $(420,970) \mathrm{GeV}$ with $A_{0}=3000 \mathrm{GeV}$ and $\tan \beta=14$, though the likelihood function is quite flat and the exact position of the best point is not very well defined.

The right panel of Fig. 4 shows that there is still hope for direct detection experiments though the new best fit point implies a cross section of $\sim 10^{-47} \mathrm{~cm}^{2}$, nearly two orders of magnitude below the current upper bound. The likelihood function, however, is rather flat between $10^{-47} \mathrm{~cm}^{2} \lesssim \sigma_{p}^{\mathrm{SI}} \lesssim$ 

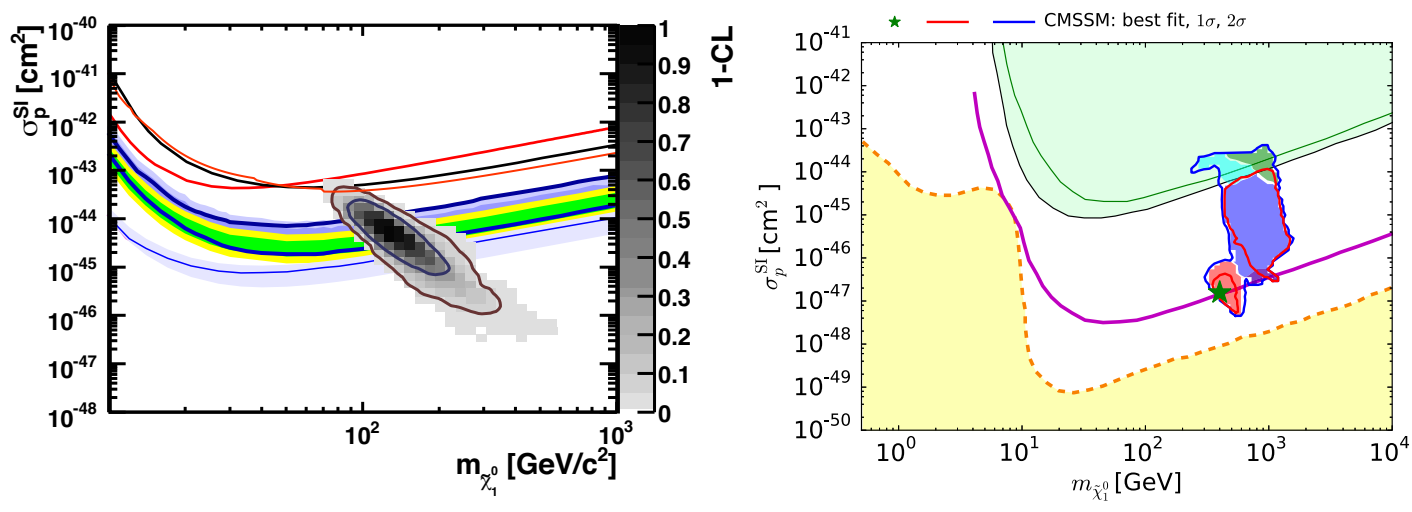

Figure 4. (left) The pre-LHC prediction for the spin-independent DM scattering cross section, $\sigma_{p}^{\mathrm{SI}}$, versus $m_{\chi}$ in the CMSSM [6]. The solid lines are the pre-LHC experimental upper limits from CDMS [10] and XENON10[11], while the bands are the more recent limits from XENON100 [12, 13] and LUX [14]. (right) The post-run I likelihood contours for $\sigma_{p}^{\text {SI }}$ [7]. Shading within the likelihood contours is the same as in Fig. 3, though here we also see a region where chargino coannihilations are dominant (green). The green and black lines show the sensitivities of the XENON100 [13] and LUX [14] experiments, respectively, and the solid purple lines show the projected $95 \%$ exclusion sensitivity of the LUX-Zeplin (LZ) experiment [15]. The dashed orange line shows the astrophysical neutrino 'floor' $[16,17]$, below which astrophysical neutrino backgrounds dominate (yellow region).

$10^{-45} \mathrm{~cm}^{2}$. Note that in this case, a lower value of $\Sigma_{\pi N}=50 \pm 7 \mathrm{MeV}$ was used. In addition to the $90 \%$ CL upper limits on $\sigma_{p}^{\text {SI }}$ given by the XENON100 and LUX experiments [13, 14], the expected reach from LZ [15] is also displayed. The level of the atmospheric neutrino background [16, 17] is shown by the shaded region at small cross sections.

As a result of the constraints imposed by the LHC searches, parameter regions compatible with the Planck determination of the relic density, are largely found in 'strips' of the parameter space, due to necessary relations in the sparticle mass spectrum. For parameters where the stau and LSP are nearly degenerate, we obtain the stau co-annihilation strip [19], or when the stop and LSP are nearly degenerate at large $A_{0} / m_{0}$, we find a stop co-annihilation strip [20]. At large $\tan \beta$, the funnel strips [21] appear when $2 m_{\chi} \simeq m_{H, A}$, where $m_{H, A}$ are the masses of the heavy Higgs scalar and pseudoscalar. Finally, there is also the possibility of a focus point region [22], where the value of the $\mu$ term becomes relatively small near the edge of where radiative electroweak symmetry breaking is possible.

An example showing the stau and stop coannihilation strips is found in left panel of Fig. 5 which shows the $\left(m_{0}, m_{1 / 2}\right)$ plane for fixed $\tan \beta=20$ and $A_{0}=2.3 m_{0}$ [20]. In the dark red shaded region at small $m_{0}$, the lighter stau is the LSP and that region is excluded. Along the border of that region, the stau and lightest neutralino are degenerate. The stau coannihilation strip tracks that boundary up to roughly $m_{1 / 2}=1 \mathrm{TeV}$ and is shown as a barely visible blue shaded strip. Along the strip, the Higgs mass (shown by the red dot dashed curves) does not exceed $124 \mathrm{GeV}$. The stop coannihilation strip is also seen in the left panel of Fig. 5. The stop strip corresponds to the thin blue line which tracks the dark red wedge in the lower right of the panel. This strip extends past $m_{0}=10 \mathrm{TeV}$. The current and future reach of the LHC is shown by the solid black, blue, green and purple lines which are particle exclusion reaches for $B_{T}$ searches with $20 / \mathrm{fb}$ at $8 \mathrm{TeV}, 300$ and $3000 / \mathrm{fb}$ at $14 \mathrm{TeV}$, and 3000/fb at a prospective HE-LHC at $33 \mathrm{TeV}$, respectively [20]. Unlike the stau strip, it is unlikely that the entire strip will be fully probed as it is seen to extend beyond the reach of a future $33 \mathrm{TeV}$ LHC upgrade. 

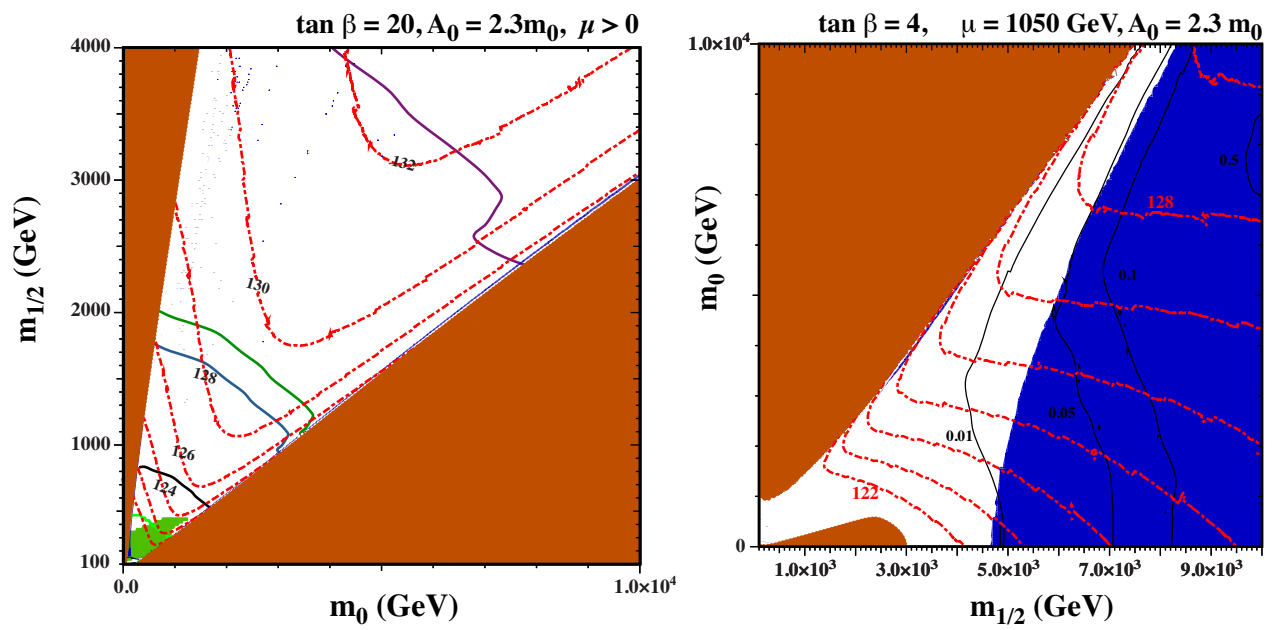

Figure 5. (left) The $\left(m_{0}, m_{1 / 2}\right)$ plane for fixed $\tan \beta=20$ and $A_{0}=2.3 m_{0}$ in the CMSSM. (right) The $\left(m_{1 / 2}, m_{0}\right)$ plane for fixed $\tan \beta=4, \mu=1050 \mathrm{GeV}$, and $A_{0}=2.3 m_{0}$. The dark red shaded regions are excluded because of a charged LSP and/or a tachyon, and the green region are excluded by $b \rightarrow s \gamma$ decay. In the dark blue strips and region (in the right panel) the relic LSP density lies within the range allowed by cosmology, and the dashed red lines are contours of $m_{h}$. The solid black, blue, green and purple lines in each panel are particle exclusion reaches for $B_{T}$ searches with the LHC at $8 \mathrm{TeV}, 300$ and 3000/fb with $\mathrm{LHC}$ at $14 \mathrm{TeV}$, and 3000/fb with HE-LHC at $33 \mathrm{TeV}$, respectively. The solid black curves in the right panel correspond to contours of constant proton lifetime in units of $10^{35} \mathrm{yrs}$.

If one goes beyond the CMSSM, dark matter regions are no longer confined to strips as seen in the right panel of Fig. 5 which shows the $\left(m_{1 / 2}, m_{0}\right)$ plane for fixed $\tan \beta=4, \mu=1050 \mathrm{GeV}$, and $A_{0}=2.3 m_{0}$ [23]. Here, the two Higgs soft masses are no longer constrained to equal the sfermion masses, $m_{0}$, though they are constrained to equal each other, thus creating a one-parameter extension of the CMSSM, known as NUHM1 [24]. In this model, one may choose $\mu$ as the free parameter in lieu of the Higgs soft masses. Here, we see a large region where relic density is in agreement with Planck. The LSP is bino-like to the left of the blue region and the relic density is too high except for the extremely thin strips along the stop (higher $m_{0}$ ) and stau (lower $m_{0}$ ) LSP areas. In this case, since $\mu$ is fixed, as the gaugino mass is increased, the LSP becomes more Higgsino-like, however asymptotically, at large $m_{1 / 2}$, the Higgsino mass tends toward $\simeq 1.1 \mathrm{TeV}$ where Higgsinos provide the correct dark matter density (this is of course tied to the choice of $\mu=1050 \mathrm{GeV}$ ) [25]. Indeed, this region would extend infinitely far to the right. As one can see, there is a significant area where the Higgsino provides the correct relic density with an acceptable Higgs mass. Also shown in this panel are contours of constant proton lifetime (in units of $10^{35} \mathrm{yrs}$ ) assuming a minimal SU(5) GUT [23]. The experimental limit would exclude points to the left of the curve labelled 0.05 or require a non-minimal GUT for which the calculated lifetime satisfies the bound.

\section{SO(10) GUT Dark Matter}

In addition to gauge coupling unification, the stability of the gauge hierarchy, and the presence of a dark matter candidate as noted earlier, supersymmetry is often motivated by the stability of the 
electroweak vacuum [26] and radiative electroweak symmetry breaking [27]. With the exception of the hierarchy problem, non-supersymmetric $\mathrm{SO}(10)$ GUT $[28,29]$ models may contain equivalences of all of these desirable features. As discussed above, in models with an intermediate scale between the electroweak scale and the GUT scale, gauge coupling unification becomes possible $[29,30]$ when the intermediate scale is determined by the unification conditions given a field content below the GUT scale as seen in Fig. 2.

To construct an SO(10) dark matter model, we should first pick an intermediate scale gauge group and a representation for the Higgs field, $R_{1}$ which breaks $\mathrm{SO}(10)$. For example, to obtain $S U(4)_{C} \otimes$ $S U(2)_{L} \otimes S U(2)_{R}$, we should take $R_{1}=\mathbf{2 1 0}$. If the intermediate scale is broken by a Higgs in a $\mathbf{1 2 6}$ representation, a residual $Z_{2}$ discrete symmetry survives enabling the possibility of a stable dark matter candidate [5,31-33]. Furthermore, in models with gauge coupling unification and a stable dark matter candidate, it is also possible to stabilize the electroweak vacuum while at the same time radiatively break the electroweak symmetry [34]. The coupling of the $\mathbf{1 2 6}$ to SM matter fields embedded in a 16 representation of $S O(10)$ naturally gives rise to a majorana mass mass to the $v_{R}$ component of the 16 of order $\langle\mathbf{1 2 6}\rangle \sim M_{\text {int }}$ which when combined with the Dirac mass arising from the vev of the SM Higgs (now residing in a 10-plet of $\mathrm{SO}(10)$ ) gives rise to the seesaw mechanism for light neutrino masses [35].

The dark matter in $\mathrm{SO}(10)$ models may be either fermionic or bosonic. A fermionic DM candidate should be parity even and belong to a 10, 45, 54, 120, 126, 210 or $\mathbf{2 1 0}^{\prime}$ representation, while scalar DM is parity odd and belongs to a $\mathbf{1 6}$ or $\mathbf{1 4 4}$ representation.

\section{Summary}

It is becoming apparent that recent LHC searches for supersymmetry have pushed CMSSM into corners of the parameter space which rely on the near degeneracy between the LSP and the next lightest superpartner, thus allowing coannihilations to reign in the relic density. While the stau coannihilation strip is nearly ruled out by LHC searches, possibilities remain for the stop strip and if there are non-universal gaugino masses, gluino coannihilation. It is also possible that $m_{0}$ is large near the focus point strip so that the LSP is mostly Higgsino-like. There are several variants of the CMSSM which still permit neutralino dark matter. These include models with non-universal Higgs scalar masses (NUHM), models where the input universality scale is below the GUT scale (subGUT models), or pure gravity mediated models with either wino or Higgsino dark matter.

While supersymmetry has many motivations beyond dark matter, with the exception of the hierarchy problem, almost of these motivating factors can be resolved in non-supersymmetric versions of $\mathrm{SO}(10)$ grand unification. The real challenge lies in the detection of dark matter and our ability to discriminate between the various models.

\section{References}

[1] John R. Ellis, S. Kelley and Dimitri V. Nanopoulos, Phys. Lett. B249, 441, 1990; John R. Ellis, S. Kelley and Dimitri V. Nanopoulos, Phys. Lett. B260, 131, 1991; Ugo Amaldi, Wim de Boer, and Hermann Furstenau. Phys. Lett., B260, 447, 1991; Paul Langacker and Ming-xing Luo, Phys. Rev. D44, 817, 1991; C. Giunti, C. W. Kim and U. W. Lee, Mod. Phys. Lett. A6, 1745, 1991.

[2] L. Maiani, in Proceedings, Gif-sur-Yvette Summer School On Particle Physics, 1979, 1-52; Gerard 't Hooft and others (eds.), Recent Developments in Gauge Theories, Proceedings of the Nato Advanced Study Institute, Cargese, France, August 26 - September 8, 1979, Plenum press, New York, USA, 1980, Nato Advanced Study Institutes Series: Series B, Physics, 59.; Edward Witten, Phys. Lett. B105, 267, 1981. 
[3] H. Goldberg, Phys. Rev. Lett. 50 (1983) 1419; J. Ellis, J. Hagelin, D. Nanopoulos, K. Olive and M. Srednicki, Nucl. Phys. B 238 (1984) 453.

[4] R. Barbieri, S. Ferrara and C. A. Savoy, Phys. Lett. B 119, 343 (1982); J. R. Ellis, K. A. Olive, Y. Santoso and V. C. Spanos, Phys. Lett. B 573 (2003) 162 [arXiv:hep-ph/0305212], and Phys. Rev. D 70 (2004) 055005 [arXiv:hep-ph/0405110].

[5] Y. Mambrini, K. A. Olive, J. Quevillon and B. Zaldivar, Phys. Rev. Lett. 110, no. 24, 241306 (2013) [arXiv:1302.4438 [hep-ph]].

[6] O. Buchmueller et al., Eur. Phys. J. C 64, 391 (2009) [arXiv:0907.5568 [hep-ph]].

[7] O. Buchmueller et al., Eur. Phys. J. C 74 (2014) 2922 [arXiv:1312.5250 [hep-ph]]; O. Buchmueller et al., Eur. Phys. J. C 74 (2014) 12, 3212 [arXiv:1408.4060 [hep-ph]]; E. A. Bagnaschi et al., Eur. Phys. J. C 75, 500 (2015) [arXiv:1508.01173 [hep-ph]].

[8] John R. Ellis, Giovanni Ridolfi and Fabio Zwirner, Phys. Lett. B257, 83, 1991; John R. Ellis, Giovanni Ridolfi and Fabio Zwirner, Phys. Lett. B262, 477, 1991; Y. Okada, Masahiro Yamaguchi and T. Yanagida, Phys. Lett. B262, 54, 1991; Yasuhiro Okada, Masahiro Yamaguchi and Tsutomu Yanagida, Prog. Theor. Phys. 85, 1, 1991; Howard E. Haber and Ralf Hempfling, Phys. Rev. Lett. 66, 1815, 1991.

[9] J. R. Ellis, D. V. Nanopoulos, K. A. Olive and Y. Santoso, Phys. Lett. B 633, 583 (2006) [arXiv:hep-ph/0509331].

[10] Z. Ahmed et al. [CDMS Collaboration], Phys. Rev. Lett. 102, 011301 (2009) [arXiv:0802.3530 [astro-ph]].

[11] J. Angle et al. [XENON Collaboration], Phys. Rev. Lett. 100, 021303 (2008) [arXiv:0706.0039 [astro-ph]].

[12] E. Aprile et al. [XENON100 Collaboration], Phys. Rev. Lett. 107, 131302 (2011) [arXiv:1104.2549 [astro-ph.CO]].

[13] E. Aprile et al. [XENON100 Collaboration], Phys. Rev. Lett. 109, 181301 (2012) [arXiv:1207.5988 [astro-ph.CO]].

[14] D. S. Akerib et al. [LUX Collaboration], Phys. Rev. Lett. 112, 091303 (2014) [arXiv:1310.8214 [astro-ph.CO]].

[15] D. C. Malling, D. S. Akerib, H. M. Araujo, X. Bai, S. Bedikian, E. Bernard, A. Bernstein and A. Bradley et al., arXiv:1110.0103 [astro-ph.IM].

[16] J. Billard, L. Strigari and E. Figueroa-Feliciano, Phys. Rev. D 89, no. 2, 023524 (2014) [arXiv:1307.5458 [hep-ph]];

[17] P. Cushman et al., arXiv:1310.8327 [hep-ex].

[18] G. Aad et al. [ATLAS Collaboration], JHEP 1409 (2014) 176 [arXiv:1405.7875 [hep-ex]]; JHEP 1510, 054 (2015) [arXiv:1507.05525 [hep-ex]].

[19] M. Citron, J. Ellis, F. Luo, J. Marrouche, K. A. Olive and K. J. de Vries, Phys. Rev. D 87, no. 3, 036012 (2013) [arXiv:1212.2886 [hep-ph]], and references therein.

[20] J. Ellis, K. A. Olive and J. Zheng, Eur. Phys. J. C 74 (2014) 2947 [arXiv:1404.5571 [hep-ph]], and references therein; O. Buchmueller, M. Citron, J. Ellis, S. Guha, J. Marrouche, K. A. Olive, K. de Vries and J. Zheng, Eur. Phys. J. C 75, no. 10, 469 (2015) [arXiv:1505.04702 [hep-ph]].

[21] M. Drees and M. M. Nojiri, Phys. Rev. D 47 (1993) 376 [arXiv:hep-ph/9207234]; H. Baer and M. Brhlik, Phys. Rev. D 53 (1996) 597 [arXiv:hep-ph/9508321]; Phys. Rev. D 57 (1998) 567 [arXiv:hep-ph/9706509]; H. Baer, M. Brhlik, M. A. Diaz, J. Ferrandis, P. Mercadante, P. Quintana and X. Tata, Phys. Rev. D 63 (2001) 015007 [arXiv:hep-ph/0005027]; J. R. Ellis, T. Falk, G. Ganis, K. A. Olive and M. Srednicki, Phys. Lett. B 510 (2001) 236 [arXiv:hep-ph/0102098]. 
[22] J. L. Feng, K. T. Matchev and D. Sanford, Phys. Rev. D 85, 075007 (2012) [arXiv:1112.3021 [hep-ph]]; P. Draper, J. Feng, P. Kant, S. Profumo and D. Sanford, Phys. Rev. D 88, 015025 (2013) [arXiv:1304.1159 [hep-ph]].

[23] J. Ellis, J. L. Evans, F. Luo, N. Nagata, K. A. Olive and P. Sandick, Eur. Phys. J. C 76, no. 1, 8 (2016) [arXiv:1509.08838 [hep-ph]].

[24] H. Baer, A. Mustafayev, S. Profumo, A. Belyaev and X. Tata, Phys. Rev. D 71, 095008 (2005) [arXiv:hep-ph/0412059]; H. Baer, A. Mustafayev, S. Profumo, A. Belyaev and X. Tata, JHEP 0507 (2005) 065, hep-ph/0504001; J. R. Ellis, K. A. Olive and P. Sandick, Phys. Rev. D 78, 075012 (2008) [arXiv:0805.2343 [hep-ph]].

[25] K. A. Olive and M. Srednicki, Phys. Lett. B 230, 78 (1989); K. A. Olive and M. Srednicki, Nucl. Phys. B 355, 208 (1991).

[26] John R. Ellis and Douglas Ross, Phys. Lett. B 506, 331, 2001, hep-ph/0012067.

[27] L. E. Ibanez and G. G. Ross, Phys. Lett. B 110, 215 (1982); K. Inoue, A. Kakuto, H. Komatsu and S. Takeshita, Prog. Theor. Phys. 68, 927 (1982) [Erratum-ibid. 70, 330 (1983)] [Prog. Theor. Phys. 70, 330 (1983)]; L. E. Ibanez, Phys. Lett. B 118, 73 (1982); J. R. Ellis, D. V. Nanopoulos and K. Tamvakis, Phys. Lett. B 121, 123 (1983); J. R. Ellis, J. S. Hagelin, D. V. Nanopoulos and K. Tamvakis, Phys. Lett. B 125, 275 (1983); L. Alvarez-Gaume, J. Polchinski and M. B. Wise, Nucl. Phys. B 221, 495 (1983).

[28] H. Georgi, AIP Conf. Proc. 23, 575 (1975); H. Fritzsch and P. Minkowski, Annals Phys. 93, 193 (1975); M. S. Chanowitz, J. R. Ellis and M. K. Gaillard, Nucl. Phys. B 128, 506 (1977); H. Georgi and D. V. Nanopoulos, Nucl. Phys. B 155, 52 (1979).

[29] H. Georgi and D. V. Nanopoulos, Nucl. Phys. B 159, 16 (1979); C. E. Vayonakis, Phys. Lett. B 82, 224 (1979) [Phys. Lett. 83B, 421 (1979)].

[30] A. Masiero, Phys. Lett. B 93, 295 (1980); Q. Shafi, M. Sondermann and C. Wetterich, Phys. Lett. B 92, 304 (1980); F. del Aguila and L. E. Ibanez, Nucl. Phys. B 177, 60 (1981); R. N. Mohapatra and G. Senjanovic, Phys. Rev. D 27, 1601 (1983); M. Fukugita and T. Yanagida, In *Fukugita, M. (ed.), Suzuki, A. (ed.): Physics and astrophysics of neutrinos* 1-248. and Kyoto Univ. - YITP-K1050 (93/12,rec.Feb.94) 248 p. C.

[31] M. Kadastik, K. Kannike and M. Raidal, Phys. Rev. D 80 (2009) 085020 [Erratum-ibid. D 81 (2010) 029903] [arXiv:0907.1894 [hep-ph]]; M. Kadastik, K. Kannike and M. Raidal, Phys. Rev. D 81, 015002 (2010) [arXiv:0903.2475 [hep-ph]]; M. Frigerio and T. Hambye, Phys. Rev. D 81 (2010) 075002 [arXiv:0912.1545 [hep-ph]].

[32] Y. Mambrini, N. Nagata, K. A. Olive, J. Quevillon and J. Zheng, Phys. Rev. D 91, no. 9, 095010 (2015) [arXiv:1502.06929 [hep-ph]].

[33] N. Nagata, K. A. Olive and J. Zheng, JHEP 1510, 193 (2015) [arXiv:1509.00809 [hep-ph]].

[34] Y. Mambrini, N. Nagata, K. A. Olive and J. Zheng, arXiv:1602.05583 [hep-ph].

[35] P. Minkowski, Phys. Lett. B 67 (1977) 421; M. Gell-Mann, P. Ramond and R. Slansky, in Supergravity, eds. D. Freedman and P. Van Nieuwenhuizen (North Holland, Amsterdam, 1979), pp. 315-321. ISBN 044485438x; T. Yanagida, in Proceedings of the Workshop on the Unified Theory and The Baryon Number of the Universe, eds O. Sawada and S. Sugamoto. KEK79-18 (1979); R. N. Mohapatra and G. Senjanovic, Phys. Rev. Lett. 44, 912 (1980); J. Schechter and J. W. F. Valle, Phys. Rev. D 22 (1980) 2227; J. Schechter and J. W. F. Valle, Phys. Rev. D 25 (1982) 774. 